\title{
Editorial
}

\section{Chemical EOR}

\author{
Ibnelwaleed A. Hussein, ${ }^{1}$ Yujun Feng, ${ }^{2}$ and Baojun Bai ${ }^{3}$ \\ ${ }^{1}$ Polymers \& Rheology Research Laboratory, Department of Chemical Engineering, King Fahd University of Petroleum \& Minerals, \\ Dhahran 31261, Saudi Arabia \\ ${ }^{2}$ Polymer Research Institute, State Key Laboratory of Polymer Materials Engineering, Sichuan University, Chengdu 610065, China \\ ${ }^{3}$ Petroleum Engineering, Missouri University of Science and Technology, Rolla, MO 65409, USA
}

Correspondence should be addressed to Ibnelwaleed A. Hussein; ihussein@kfupm.edu.sa

Received 15 December 2013; Accepted 15 December 2013

Copyright @ 2013 Ibnelwaleed A. Hussein et al. This is an open access article distributed under the Creative Commons Attribution License, which permits unrestricted use, distribution, and reproduction in any medium, provided the original work is properly cited.

Enhanced oil recovery (EOR) has become more and more important due to the growing energy demand and depleting oil reserves. Among the various EOR methods, chemical EOR was considered to be expensive and not economic when oil price was below $\$ 40$ and field applications have almost completely stopped during that time except in China, where around 13 million tons of oil is produced additionally per year by these chemical flooding techniques. However, chemical EOR has recently drawn increasing interest due to the continuous high oil prices within the last 7 years and the scarcity of cheap oil reserves. Therefore, the chemical EOR research has shown major growth in the recent years.

This special issue is on chemical EOR systems. It reports some results of research, pilot tests, and field applications related to chemical EOR. The focus is on synthesis and laboratory testing as well as field applications of new chemical EOR materials used in flooding processes. These manuscripts covered a broad spectrum of topics ranging from synthesis of new polymers for EOR applications to both onshore and offshore field applications of commercially available polymers and foams systems. The subject of designing new systems for high-temperature, high-salinity reservoir environment is also investigated. The chemical systems reported in these papers can be used to reduce interfacial tension, increase injection fluid viscosity, and reduce the permeability of highpermeability streaks. They can increase microscomic displacement efficiency and/or sweep efficiency, thus improving the oil recovery.

\section{Acknowledgments}

Finally, we would like to thank the authors who contributed to this special issue. Special thanks are due to the reviewers for their help in evaluating the submitted papers. We hope that this special issue on chemical EOR will help the readers in advancing their knowledge in such an important field.

\section{Ibnelwaleed A. Hussein Yujun Feng Baojun Bai}



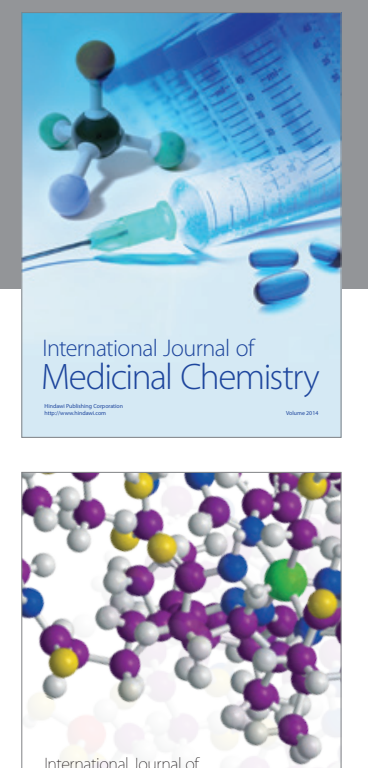

\section{Carbohydrate} Chemistry

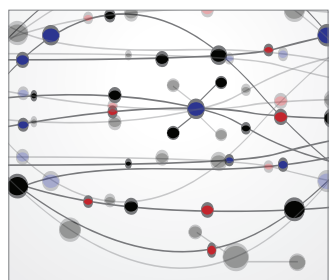

The Scientific World Journal
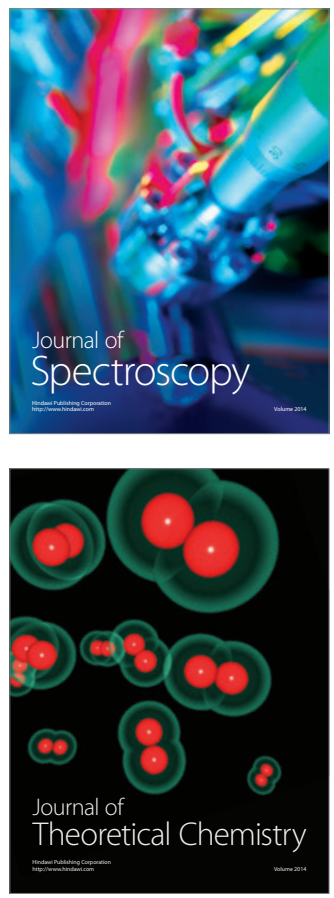
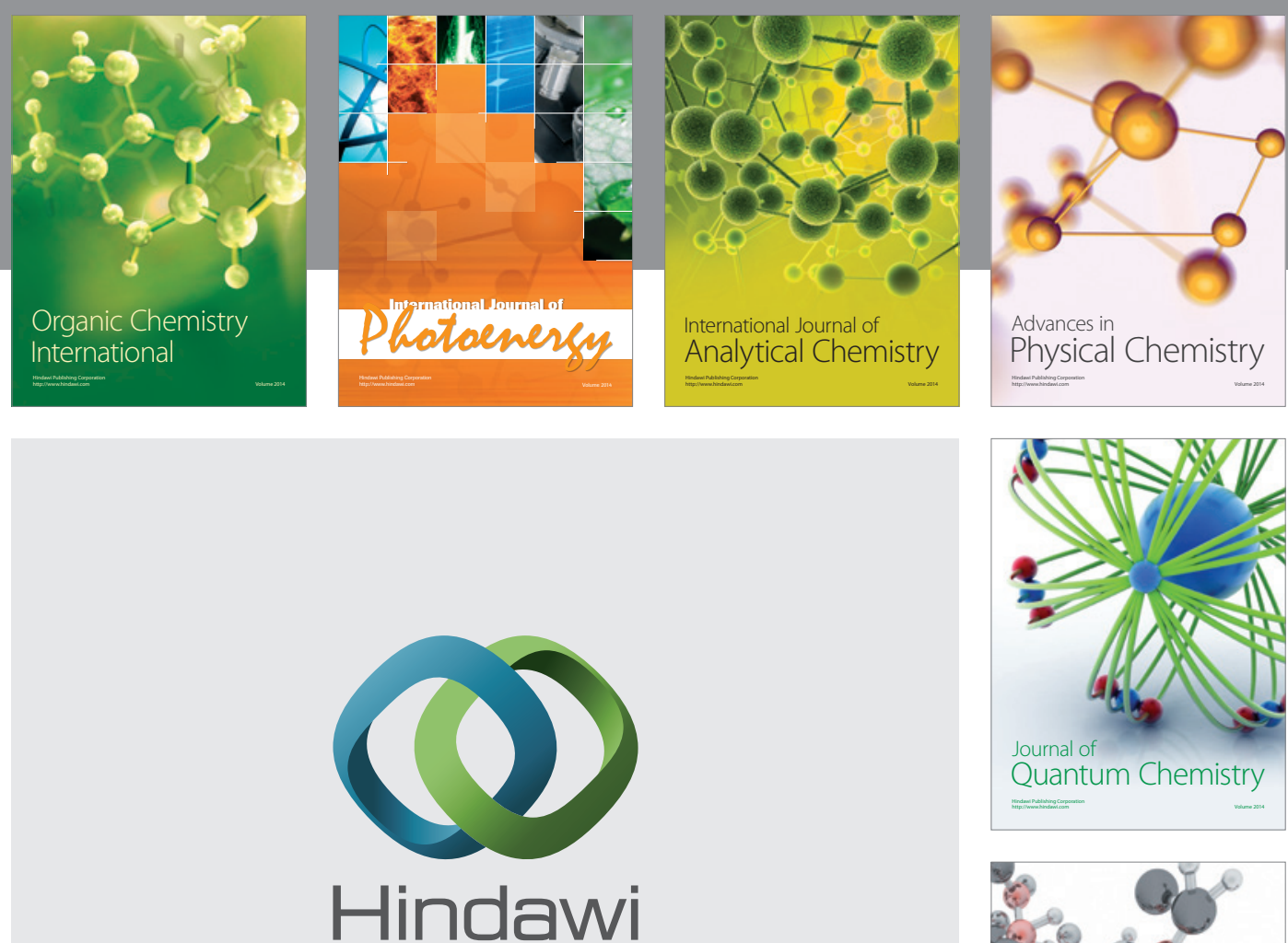

Submit your manuscripts at

http://www.hindawi.com

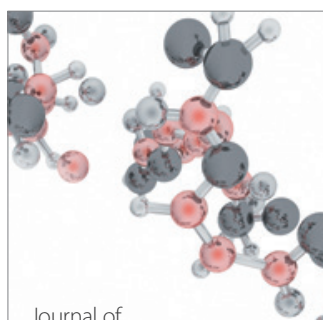

Analytical Methods

in Chemistry

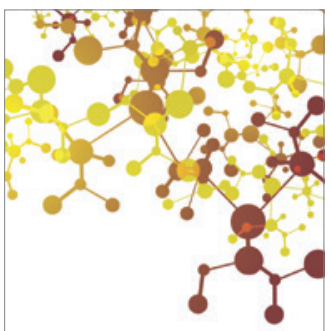

Journal of

Applied Chemistry

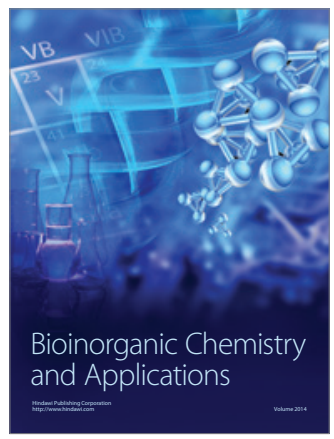

Inorganic Chemistry
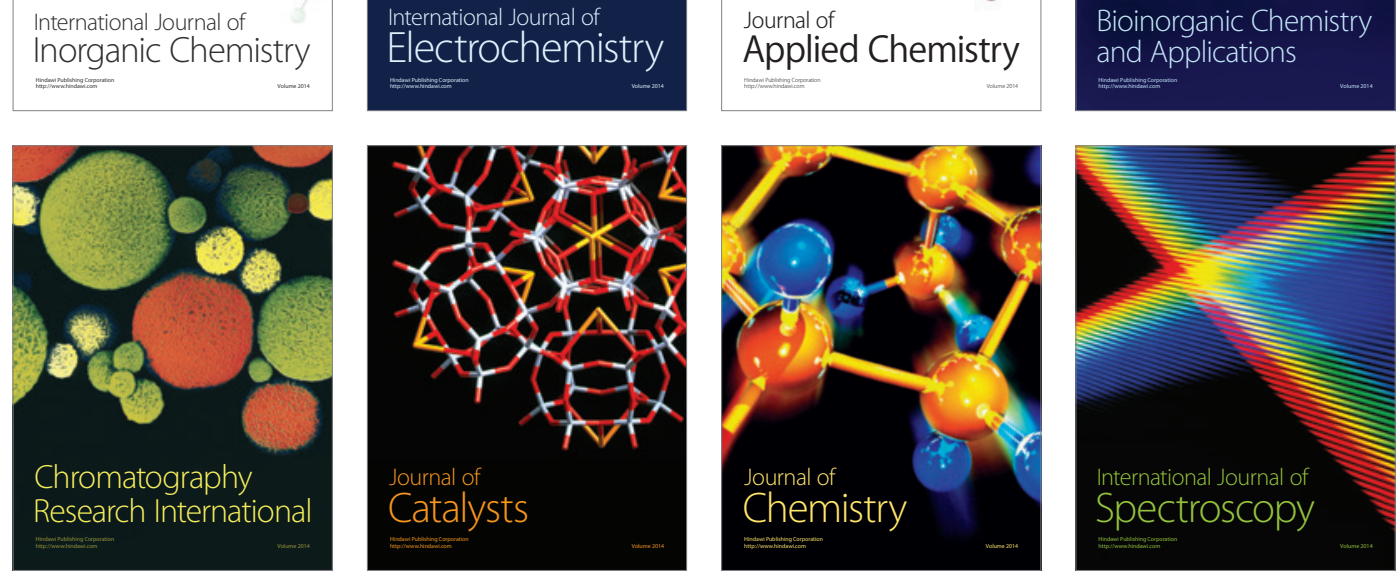